\title{
In Vivo Estimation of Coronary Mechanical Wall Deformation and its Relation to Plaque Formation
}

\author{
M Roberts, SP Corney, A Thomson, J Bashford, AG Kan \\ School of Medicine, University of Tasmania, Australia
}

\begin{abstract}
When a coronary artery moves, the wall has to change shape. Thus in each cardiac cycle some regions of the wall contract and some dilate. The stress on the coronary wall during ventricular contractions can be calculated by tracking elements in the arterial wall throughout the cycle and calculating their deformation. The method employs a $3 D$ reconstruction process to generate model input for a structural mechanical finite elements package. The reconstruction algorithm is applied to biplane angiogram images taken at different stages of the cardiac cycle and the data fed into a package to generate the corresponding meshed model configurations.

The calculation of conformational stress requires two further assumptions: the minimal residual stress state is selected to be the configuration just prior to systole, and secondly, a model for material properties of coronary artery walls has to be assumed. We have developed packages in Mathematica ${ }^{T M} 4.1$ for construction of meshed arteries, estimating local conformational deformation and associated stress quantities.
\end{abstract}

\section{Introduction}

It is now widely accepted that prognosis of coronary disease is inversely related to the number of severely stenotic vessels and that high-grade coronary stenosis increases the risk for future coronary occlusions. For example Moise [1] demonstrated that high-grade stenosis ( $>80 \%$ ) was the single most predictive factor for developing coronary artery occlusion. However, there have been several studies $[2,3,4]$ that have shown that degree of stenosis is a poor predictor of the location of future atheroma.

It is now becoming increasingly accepted that mechanical forces are involved in the initiation and development of atherosclerosis [5, 6]. Furthermore, studies suggest that coronary arterial motion might play an important role in the pathogenesis of coronary atherosclerosis [7, 8]. In particular [8] has noted there is a weak, but statistically significant relation, between the rate of progression of atherosclerosis and the angle of arterial flexion during systole at the plaque location. Other studies considered other geometrical properties such as radius of curvature and torsion, however we suggest a more sophisticated method of analysis which borrows stress analysis techniques from mechanical engineering and utilizes them to analyze conformational stress in the (right) coronary artery. We use our method to test the hypothesized relationship between the periodic changes in wall conformational shear stress and the progression of stenoses. In the future, we hope to determine, through longitudinal studies, whether particular conformal stress distributions may be used as an aid in predicting the progression of atheroma.

\section{Method}

This study was approved by the University of Tasmania and the Royal Hobart Hospital Human Ethics committee.

Digital biplane angiography was performed on patients using routine techniques and viewing angles. A model of the right coronary artery is generated from a pair of non-coplanar angiogram images via a two-step process. Firstly, following the procedure developed in $[9,10,11]$, three-dimensional coordinates of points along the artery midline are calculated in addition to estimates of the inner radii at these locations. The resulting data are used as input for a cubic spline interpolation routine written in Mathematica ${ }^{T M} 4.1$, to generate a model artery with elliptical cross-section. Commencing with the minimal stress state, which is taken to be the frame just prior to systole, the relative conformal displacement of each mesh element in each frame is determined. Assuming a stress-strain relationship for the intima the conformal stress invariant is then calculated.

\subsection{Reconstruction}

The technique for taking a pair of biplane images and converting to a list of Cartesian coordinates $(x, y, z)$ of points along the artery midline plus estimates $(a, b)$ of the internal radii is detailed in $[9,11]$. In summary, the procedure involves determining the centreline and radii in each image and by using the relative viewing angles, as included in the DICOM format, a point in one plane can 


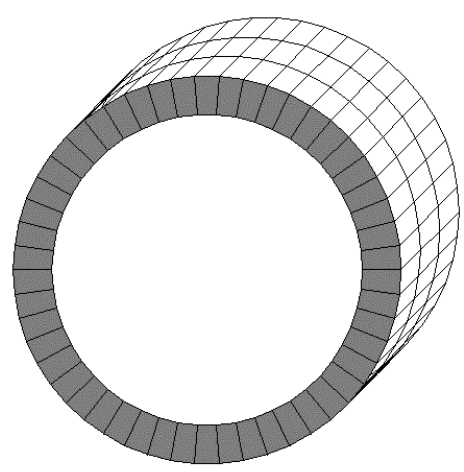

Figure 1. A close up of the mesh, showing the annular structure.

be mapped on to a line in the second. If the first point is on the artery then the intersection of the line and artery in the second image is the corresponding point. The set of these pairs of points can then be combined to give the centreline in three dimensions.

By picking at least three fiducial points that can be accurately determined in both images, we can use a least squares algorithm to compensate for any errors in the viewing angles, and also for the non-isocentricity of the artery.

In order to accurately model the artery the centreline is reconstructed on a pixel by pixel basis, which we then smooth to typically $30-50$ points for the combined proximal and mid sections of a right coronary artery.

\subsection{Mesh generation}

We define a single layer annular mesh that consists of 50 hexahedral elements circumferentially and in the order of 300 elements along the artery. Figure 1 shows a schematic of this mesh.

For a mesh of hexahedral volume elements $H_{i j k}$, each element contains eight nodes $N_{p m n}$. It is then convenient to label each element in terms of the nodes comprising its vertices

$$
\begin{aligned}
H_{i j k}= & \left(N_{i, j, k}, N_{i+1, j, k}, N_{i+1, j, k+1}, N_{i, j, k+1}, N_{i, j+1, k},\right. \\
& \left.N_{i+1, j+1, k}, N_{i+1, j+1, k+1}, N_{i, j+1, k+1}\right), \\
\equiv & H_{i j k \ell} ; \quad \ell, 1 \ldots 8
\end{aligned}
$$

\subsection{Frame matching}

Not all types of arterial motion can be studied using this method, for example 'twisting' in the circumferential direction and radial shear cannot be monitored, and there is only a limited ability to measure elongation/compression along the arterial axis. The movement that we shall primarily be studying is deformation (c.f. 'flexion', [8]) caused by the change in the axial curvature associated with shifts in conformation during the cardiac cycle.

Close to junctions the relative motion and rigidity constraints on the three branches will cause circumferential twisting. While estimates of orientation shift between adjacent junctions in successive frames could, in principle, be made there is no guarantee of their accuracy. Study of the twisting and radial components, the latter of which is typically small, would be better suited to imaging techniques where data of the full arterial surface is available, for example angiography coupled with IVUS.

In order to calculate the stress, each element is compared to the corresponding point in the zero-stress mesh, thus an accurate method of matching the corresponding elements in each mesh is required.

When matching centerlines between the unstressed frame $F_{0}$ and the stressed frame $F_{i}$, two difficulties arise. Firstly, that the starting point in each frame, though similar, usually only matches within $<3$ millimeters, secondly, the artery during diastole elongates by as much as $20 \%$. We denote a centerline point on $F_{0}$ that is a distance $x$ from the top of $F_{0}$ as $P_{x}$, and similarly $Q_{x}$ for a point on $F_{i}$. We can calculate, in the spirit of [12], the 'directional distance' between $P$ and the point $Q$ in $F_{i}$ according the the modified norm,

$$
\mathrm{rms}_{\text {mismatch }}=\sqrt{\frac{1}{\mathrm{~N}} \sum_{\mathrm{i}=1}^{\mathrm{N}}\left\|\mathrm{P}_{\mathrm{h}+\mathrm{i}}, \mathrm{Q}_{\mathrm{h}+\mathrm{h}^{\prime}+\mathrm{ik}}\right\|^{2}}
$$

where $h \approx 1 \mathrm{~mm},\left|h^{\prime}\right| \approx 3 \mathrm{~mm}, 0.9<k<$ 1.25 (elongation),

$$
\|P, Q\|=\frac{\sqrt{\left(P_{x}-Q_{x}\right)^{2}+\left(P_{y}-Q_{y}\right)^{2}+\left(P_{z}-Q_{z}\right)^{2}}}{\left|\hat{v}_{p} \cdot \hat{v}_{q}\right|}
$$

and

$$
\hat{v}_{p} \cdot \hat{v}_{q} \text { are the unit tangent vectors at } P \text { and } Q \text { resp. }
$$

We match points in different frames by minimising $\mathrm{rms}_{\text {mismatch }}$ along the length of the artery.

We note that the dot product is equal to the cosine of the angle between the tangent vectors at $P$ and $Q$ respectively.

\subsection{Displacement estimation}

For each vertex of a stressed element, we define the conformal displacement relative to the corresponding vertex in the unstressed element as the displacement that cannot be accounted for by overall elemental translations or rotations.

Once a volume element in the undeformed mesh has been identified with an element in a deformed mesh, 
estimates of the node displacements are readily made with the (necessary) assumption that no circumferential twisting has occurred. To minimise errors, nodes common to neighbouring elements are averaged. Thus, for example, for $i, j, k>1$

$$
\begin{aligned}
H_{i, j, k, 1} & =H_{i, j, k-1,2}=H_{i-1, j, k-1,3}=H_{i-1, j, k, 4}= \\
H_{i, j-1, k, 5} & =H_{i, j-1, k-1,6}=H_{i-1, j-1, k-1,7}=H_{i-1, j-1, k}
\end{aligned}
$$

The three displacement fields of a hexahedral element vertex $H_{i, j, k, \ell}=(\ell, 1 \ldots 8)$ are assumed to be of the form

$$
\begin{aligned}
u_{\ell}(x, y, z)= & \alpha_{1}+\alpha_{2} x+\alpha_{3} y+\alpha_{4} z+\alpha_{5} x y+\alpha_{6} y z \\
& +\alpha_{7} z x+\alpha_{8} x y z \\
v_{\ell}(x, y, z)= & \beta_{1}+\beta_{2} x+\beta_{3} y+\beta_{4} z+\beta_{5} x y+\beta_{6} y z \\
& +\beta_{7} z x+\beta_{8} x y z \\
w_{\ell}(x, y, z)= & \gamma_{1}+\gamma_{2} x+\gamma_{3} y+\gamma_{4} z+\gamma_{5} x y+\gamma_{6} y z \\
& +\gamma_{7} z x+\gamma_{8} x y z .
\end{aligned}
$$

Therefore the internal displacements of a hexahedron may be represented as a 24-dimensional vector $\vec{d}$.

With the conformal displacements calculated, there are a variety of invariants that may be calculated - each of course, independent of the coordinate system used. We utilize the octahedral stress invariant, which is proportional to the first invariant of the Cauchy stress tensor, because it is this invariant that is most frequently used in evaluating the volumetric distortion and failure behaviour of materials.

\subsection{Material properties}

It has been frequently noted that angiography is only useful in determing the lumen size of the artery and not the external artery diameter. Since the advent of IVUS it has been clearly demonstated that the angiogram, as good as it is in indicating stenoses, is less sensitive to detecting plaques [13]. The reason for this is that many plaques bulge outwards rather than inwards, some do both (known as arterial remodeling or compensatory dilatation) and some only bulge inwards.

This has implications on calculations of stress and strain. There needs to be an assumption about the relationship between the inner and outer arterial diameters. There are generally three simple ones:

- The wall is of constant thickness, set in the program. Say,

$r_{\text {out }}=r_{\text {inner }}+0.5 \mathrm{~mm}$;

- The outer diameter is constant, set at, say, $r_{\text {out }}=$ $\max \left(r_{\text {inner }}\right)+0.5 \mathrm{~mm}$. That is, plaques only bulge inwardly;

- A 'typical diameter' $r_{a v}$ is calculated as $0.25 \mathrm{~mm}$ (say) more than the largest internal diameter, and then the outer is equal to $r_{\text {outer }}-r_{a v}=r_{a v}-r_{\text {inner }}$. That is the plaque extrudes outwards as much as it does inwards.
Our software is able to process the conformational stress based on any of these models. Preliminary results indicate that for healthy arteries the stress distribution for these three models are qualitatively similar. It is too early to indicate how much of an effect this has on highly-stenosed arteries.

Linear, elastic isotropic materials depend on two parameters: Young's modulus $E$ and Poisson's ration $\nu$. ${ }_{8}$ The stress-strain relation is

$$
\begin{aligned}
\vec{\sigma} & =\mathcal{E} \vec{\varepsilon}+\sigma_{0} \\
& =\mathcal{E} B \vec{d}+\sigma_{0} .
\end{aligned}
$$

where $\mathrm{B}$, the $6 \times 24$ strain displacement matrix is

$$
B(x, y, z)=\left(\begin{array}{c}
\partial_{x} u \\
\partial_{y} v \\
\partial_{z} w \\
\partial_{y} u+\partial_{x} v \\
\partial_{z} u+\partial_{x} w \\
\partial_{z} v+\partial_{y} w
\end{array}\right)
$$

$\sigma_{0}$ is the residual stress and $\mathcal{E}$ is the $6 \times 6$ Cauchy stress tensor.

Unweighted inter-element averaging was performed to smooth potential discontinuities and spread errors. The elemental octahedral stress can then be calculated as

$$
\begin{aligned}
& \tau_{o c t}= \\
& \frac{1}{3} \sqrt{\left(\sigma_{x}-\sigma_{y}\right)^{2}+\left(\sigma_{y}-\sigma_{z}\right)^{2}+\left(\sigma_{z}-\sigma_{x}\right)^{2}+6\left(\tau_{x y}^{2}+\tau_{y z}^{2}+\tau_{z x}^{2}\right)}
\end{aligned}
$$

\section{Results and discussion}

For an incompressible material we require the limit $\nu \rightarrow$ $1 / 2$, while initially for comparison with the model of Stein et al. [8], the $E$ value for both the artery wall and plaque were set to $2400 \mathrm{kPa}$. Subsequently, the $E$ value for plaque was increased tenfold [8].

We include, as prelimary observations, the relative conformational stress distributions of the right coronary artery for two patients, one healthy and one with a stenosis.

Figure 2 shows the diastolic phase of a healthy artery. The images are taken 80 milliseconds apart and cover the length of diastole. The first image is taken as the zero stress state, and thus the stress patterns shown are relative to this image. At this stage we have not yet validated our results using clinical or laboratory studies, and so much interpretation would seem premature. We note however, that this result indicates that healthy arteries do exhibit regions of significant increase in conformational stress during a cardiac cycle.

Two frames of a diseased artery are shown in figure 3. The first image is the reference, or zero stress state at the start of diastole, whilst the second is 320 milliseconds later, towards the end of diastole. The right most image shows a close up of the stenosed section where it can 

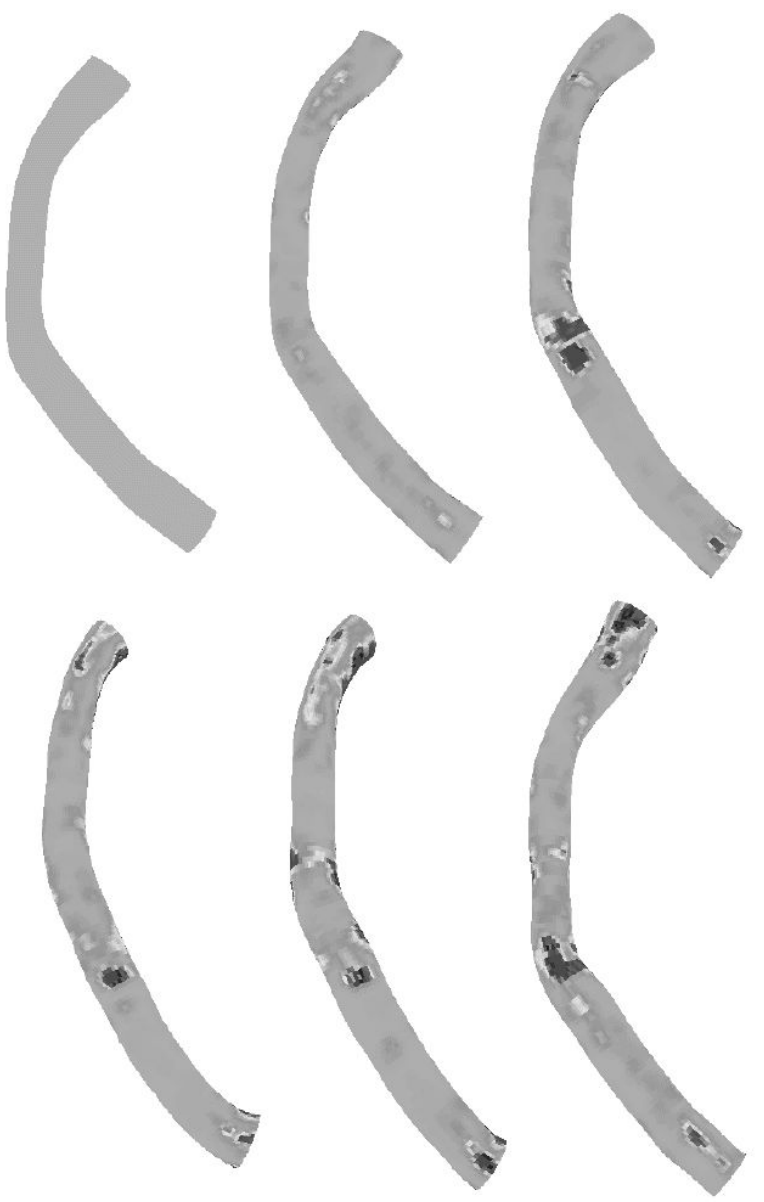

Figure 2. The relative conformational stress as calculated for a healthy artery.

be seen that the points of high conformational stress do not occur in the region of maximal stenosis, but on either side of it. If conformational stress can be used as a predictor of atheroma then this observation indicates that the atherosclerotic plaque is likely to lengthen, or possibly increase in severity, as is often seen in patients.

\section{References}

[1] Moise A, Lesperance J, Theroux P. Clinical and angiographic predictors of new total coronary occlusions in coronary artery disease. Am J Cardiol 1984;224:1176.

[2] Little W, Constantinescu M, Applegate R. Can coronary angiography predict the site of a subsequent myocardial infarction in patients with mild-to-moderate coronary artery disease? Circulation 1998;78:1157.

[3] Falk E, Prediman K, Fuster V. Coronary plaque disruption. Circulation 1995;92:657.

[4] Hackett D, Davies G, Maseri A. Pre-existing coronary stenoses in patients with first myocardial infarction are not necessarily severe. Eur Heart J 1988;9:1317.

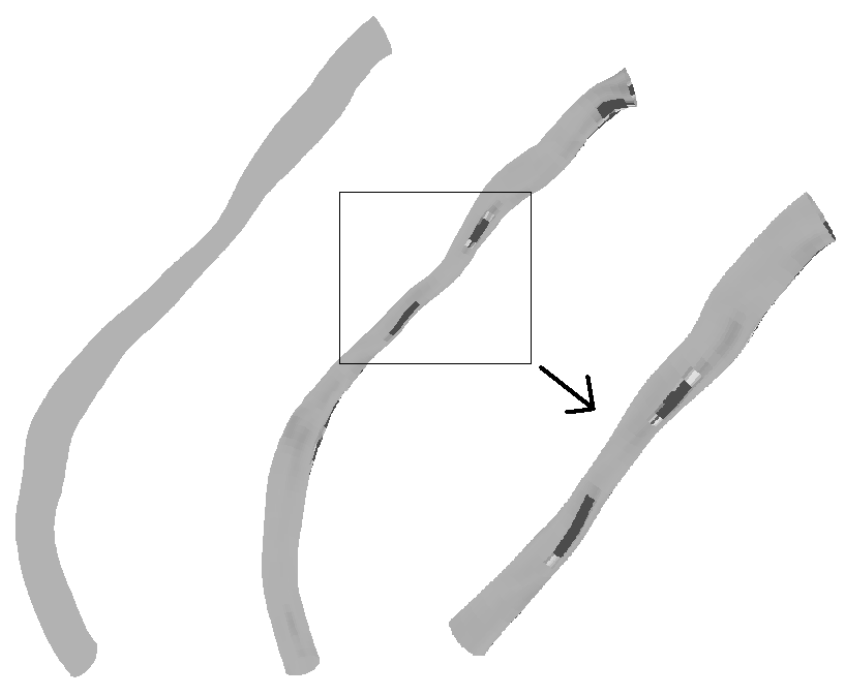

Figure 3. The relative conformational stress for two frames of a stenosed artery. The inset shows a close up of the stenosed region, showing that the higher stress exists on either side of the stenosis.

[5] Caro G, Fitzgerald J, Schroter R. Arterial wall distribution of early atheroma in man. Nature 1969;223:1159.

[6] Fung Y. Biomechanics: Circulation. Springer-Verlag, 1996.

[7] Coatrieux J, Garraeu M, Collorec R, Roux C. Computer vision approaches for the three-dimensional reconstruction of coronary arteries: review and prospects. Critical Rev Biomed Eng 1994;22:1.

[8] Stein P, Hamid M, Shivkumar K, Davis T, Khaja F, Henry J. Effects of cyclic flexion of coronary arteries on progression of atherosclerosis. Am J Cardiol 1994;73:431.

[9] Corney S, Johnston P, Kilpatrick D. Cyclic flow patterns in human coronary arteries. In Murray A (ed.), Computers in Cardiology. IEEE, 2001; 21.

[10] Corney S, Johnston P, Kilpatrick D. Modelling blood flow in coronary arteries with junctions. In Murray A (ed.), Computers in Cardiology. IEEE, 2002; 363.

[11] Corney S, Johnston P, Kilpatrick D. Construction of realistic, branched, three-dimensional arteries suitable for computational modelling of flow. submitted to Med Biol Eng Comp, 2002.

[12] Ding Z, Friedman H. Quantification of 3 dimensional coronary arterial motion using clinical biplane cineangiograms. Int J Cardiac Imaging 2000;16:331.

[13] Davies M. Coronary artery remodelling and the assessment of stenosis by pathologists. Histopathology 1998;33:497.

Address for correspondence:

Stuart Corney

School of Medicine, University of Tasmania, Australia

GPO Box 252-34 / Hobart /7001 / Australia

tel./fax: +61-3-6226-4807/4894

Stuart.Corney@utas.edu.au 